\title{
Influence of photoperiod and 6-methoxybenzoxazolinone on the reproductive axis of inbred LSH/Ss Lak male hamsters
}

\author{
H. F. Urbanski, S. O. Kim and M. L. Connolly \\ Division of Neuroscience, Oregon Regional Primate Research Center, Beaverton, Oregon 97006 , \\ $U S A$
}

\begin{abstract}
Summary. Adult male Syrian hamsters of the inbred LSH/Ss Lak strain were maintained under a 14L:10D light cycle until 13 weeks of age. At this point, they were implanted s.c. with elastomer capsules that were either empty or packed with $30-40 \mathrm{mg}$ of 6-methoxybenzoxazolinone (6-MBOA), a compound found naturally in some monocotyledonous plants; half of the animals from each treatment group were then kept in long days (14L:10D) or transferred to short days (9L:15D). Testicular size was measured and blood samples collected from each hamster immediately before capsule implantation and again 2, 4, 6 and 8 weeks later. Within just 2 weeks of exposure to short days the mean plasma levels of LH and FSH had significantly declined, in both the control and 6-MBOA-treated animals, and were basal within 4 weeks. Testicular size closely followed these gonadotrophin changes; within 4-6 weeks the testes from all of the short-day hamsters had completely regressed to a prepubertal size. At the end of the experiment, at Week 8, the animals were killed and various components of the hypothalamo-pituitary-testicular axis were compared between the treatment groups. The pituitary content of FSH and $\mathrm{LH}$, testicular weight, mean serum level of testosterone, but not hypothalamic LHRH content or pituitary gland weight, were considerably lower in the short-day than in the long-day hamsters, regardless of whether or not they had been chronically treated with 6-MBOA.

In conclusion, these results demonstrate a short-day response in male LSH/Ss Lak hamsters which is more rapid and uniform than that typically shown by outbred hamster stocks. This inbred strain is, therefore, potentially very valuable for studies of neuroendocrine mechanisms involved in mammalian photoperiodism. Additionally, although 6-MBOA can stimulate testicular growth and reproduction in microtine rodents the present results fail to demonstrate any significant effect on reproductive function in the male Syrian hamster. The study also reports on the characterization of a new conformational antiserum to LHRH which can be used in radioimmunoassays for the measurement of LHRH from three vertebrate classes (mammalian LHRH, chicken I LHRH and salmon LHRH).
\end{abstract}

Keywords: 6-MBOA; LH; FSH; LHRH; testosterone; LHRH antibody; hamster

\section{Introduction}

The Syrian or golden hamster (Mesocricetus auratus) is a seasonally breeding rodent that has been used extensively in studies of mammalian reproduction (e.g. Stetson \& Tate-Ostroff, 1981; Steger et al., 1985; Alleva, 1987). Its popularity in this regard stems from the fact that it can exploit photoperiodic information to regulate the onset and termination of its breeding season. Hamsters living in temperate regions of the world generally cease to be reproductively active during the 
autumn, as daylength decreases, and remain sexually inactive for approximately 5 months (Vendrely et al., 1971; Urbanski et al., 1983). The reproductive system of adult hamsters maintained in the laboratory can similarly be manipulated by exposure of the animals to various photoperiods (Gaston \& Menaker, 1967; Turek et al., 1975; Stetson \& Tate-Ostroff, 1981; Steger et al., 1982). Although it usually takes $8-10$ weeks of maintenance under short days for hamsters to revert from a sexually mature to a prepubertal-like condition recent observations made in female hamsters of the inbred $\mathrm{LSH} / \mathrm{Ss}$ Lak strain indicate that termination of oestrous cyclicity occurs more rapidly and uniformly than in outbred hamster stocks (Hauser \& Benson, 1986; Reiter et al., 1989). It is, however, unclear whether males from this strain also undergo a rapid short-day photoperiodic response. If true, this would make the male LSH/Ss Lak hamster a particularly valuable animal model for the study of neuroendocrine mechanisms that underlie mammalian photoperiodism. Consequently, the first aim of the present study was to determine how quickly it takes the hypothalamo-pituitary-testicular axis of $\mathrm{LSH} / \mathrm{Ss} \mathrm{Lak}$ hamsters to respond to shortening of the photoperiod. The second aim was to determine whether 6-methoxybenzoxazolinone (6-MBOA) could overcome the inhibitory effects of short days on the reproductive axis. This compound is a phenolic metabolite found in the leaves of some monocotyledonous plants and has been shown to stimulate the reproductive axis of microtine rodents, such as the mountain vole, Microtus montanus (Berger et al., 1981, 1987; Sanders et al., 1981). Its effects on the male reproductive axis of other mammals have not yet been clearly established.

\section{Materials and Methods}

Animals. Thirty-two 10-week-old male Syrian hamsters of the LSH/Ss Lak strain were obtained from Charles River (Wilmington, MA, USA) and group housed (4 animals per cage) under controlled temperature $\left(2 \mathrm{I}-22^{\circ} \mathrm{C}\right)$ and a lighting schedule that comprised $14 \mathrm{~h}$ of light per day $(14 \mathrm{~L}: 10 \mathrm{D}$; lights on $04: 00 \mathrm{~h})$. Pelleted rodent chow and water were made available at all times.

6-MBOA. The compound 6-methoxybenzoxazolinone (6-MBOA) was obtained from Chem-BioChem Research (Salt Lake City, UT, USA) and prepared for chronic in-vivo administration following a previously described protocol (Butterstein et al., 1985). Capsules were constructed from 3-cm lengths of Silastic medical-grade tubing (Dow Corning Corporation, Midland, MI, USA; 0.062" i.d., 0.125" o.d.) and packed with 30-40 mg 6-MBOA. The capsules were incubated overnight in saline $(0.9 \% \mathrm{NaCl})$, at $37^{\circ} \mathrm{C}$, before subcutaneous implantation.

Blood sampling. A sample of blood was collected from each hamster every 2 weeks. The animals were first anaesthetized with methoxyflurane (Metofane; Pitman-Moore, Inc., Washington Crossing, NJ, USA) and the thorax liberally swabbed with $70 \%$ ethanol. Next, the right external jugular vein was surgically exposed and approximately $300 \mu \mathrm{l}$ blood were drawn into a heparinized syringe fitted with a 24-gauge needle; to keep loss of blood to a minimum during the procedure the tip of the needle was passed through a small portion of pectoral muscle before penetrating the underlying vein. The wound was closed with two stainless-steel clips and the animals were given an intramuscular injection of the antibiotic, gentamicin sulphate $(0.5 \mathrm{mg})$. The samples were centrifuged and the plasmas kept frozen until assayed for LH and FSH.

Experimental design. Upon reaching 13 weeks of age the hamsters were anaesthetized with methoxyflurane and implanted with either empty or 6-MBOA-filled capsules. Half of the animals from the test and control groups $(\mathrm{N}=16)$ remained under long days while the other half $(\mathrm{N}=16)$ were transferred to short days $(9 \mathrm{~L}$ : 15D; lights on $07: 30 \mathrm{~h}$ ). Blood samples were collected from each animal immediately before capsule implantation and then 2, 4, 6 and 8 weeks later. At these time points body weight and testicular width (measured using callipers) were also recorded. Immediately following collection of the last blood sample, at Week 8, the animals were decapitated. Trunk blood was collected and the serum frozen for subsequent assay of testosterone. The testes and pituitary glands were removed and weighed. The latter were homogenized in $0.2 \mathrm{M}$-sodium phosphate buffer containing $0.9 \% \mathrm{NaCl}(\mathrm{pH} 7.6)$, frozen, and subsequently assayed for LH and FSH content. The hypothalami were also removed, homogenized in $0.1 \mathrm{M}$-acetic acid, and kept frozen until assayed for luteinizing hormone-releasing hormone (LHRH) content.

Radioimmunoassays (RIAs). Serum testosterone was measured by RIA according to a previously described protocol (Resko et al., 1973) which had an intra-assay coefficient of variation of $5 \cdot 3 \%$ and sensitivity of $10-15 \mathrm{pg} / \mathrm{ml}$. The results represent the mean value from determinations made using $10 \mu \mathrm{l}$ and $30 \mu \mathrm{l}$ serum samples after subtraction of values from water blanks (1.5 pg). RIA kits provided by the NIDDK were used to measure plasma levels and the pituitary content of LH and FSH; these comprised antigens rLH-I-6 and rFSH-I-6 for iodination and rabbit antisera rLH-S-10 and rFSH-S-11. The results are expressed in terms of the rat LH-RP-1 and FSH-RP-1 standards, respectively, both of which showed parallelism with hamster serum and pituitary extract. As little as $8 \mathrm{ng} \mathrm{LH} / \mathrm{ml}$ and $32 \mathrm{ng}$ 
$\mathrm{FSH} / \mathrm{ml}$ could be detected with these assays, which had intra-assay coefficients of variation of $11 \%$ and $9 \%$. Hypothalamic LHRH content was measured by RIA utilizing a novel LHRH antiserum, HU60. This antiserum was raised in a female New Zealand White rabbit immunized against LHRH, conjugated to bovine thyroglobulin using 1-ethyl3-[3-dimethyl-aminopropyl] carbodiimide (Sigma Chemical Co., St Louis, MO, USA). HU60 appears to recognize equally the intact mammalian decapeptide, chicken I LHRH, salmon LHRH and, to a lesser extent, [D-Phe ${ }^{2}$ ]LHRH but not $\left[\mathrm{D}-\mathrm{Ala}^{6}\right] \mathrm{LHRH}$. On the other hand, it shows no cross-reactivity with the LHRH-free acid or LHRH fragments, and only $0.5 \%$ reactivity with pro-LHRH. The antiserum therefore appears to bind almost exclusively to the mature undegraded form of the LHRH molecule. Its specificity to LHRH is highlighted by its failure to cross-react with other neuropeptides, including atrial naturetic factor, growth hormone-releasing hormone, oxytocin, somatostatin, thyrotrophin-releasing hormone, and vasoactive intestinal peptide. This antiserum was used at a final dilution of $1: 300000$ and addition of iodinated LHRH ( 8000 c.p.m.) to the assay tubes was delayed by $24 \mathrm{~h}$ to increase sensitivity. The bound LHRH was precipitated on the third day of incubation using $1 \mathrm{ml}$ cold ethanol and pelleted by centrifugation ( $30 \mathrm{~min}$ at $2000 \mathrm{~g}$ ). After aspirating the supernatants the sample radioactivity was measured using a gamma spectrometer. This RIA system had an intra-assay coefficient of variation of $12 \%$ and was able to detect as little as $0 \cdot 2 \mathrm{pg}$ LHRH per $150 \mu \mathrm{l}$ sample. Using HU60, curves derived from dilutions of homogenized hypothalamic pools showed parallelism with the LHRH standard curve while pre-immune serum from the same rabbit (at 1:300 000 dilution) completely failed to bind LHRH.

Statistics. The results are expressed in terms of group means ( $\mathrm{N}=8$ per group). Data obtained from the same animals at 2-week intervals were analysed by analysis of variance, followed by the Newman-Keuls multiple range test. Student's $t$ test was used to compare reproductive parameters between the two photoperiodic groups and also between the 6-MBOA treatment groups and the appropriate controls.

\section{Results}

The control hamsters which were maintained continuously under long days showed a gradual, but significant, increase $(P<0.01)$ in body weight (from $116 \pm 3.9 \mathrm{~g}$ at Week 0 to $142 \pm 4.4 \mathrm{~g}$ at Week $8)$. In contrast, the control hamsters that were transferred to short days failed to show any significant changes (Fig. 1). Under both photoperiods, however, there was no difference in body weight between the 6-MBOA-treated and control animals. In long-day hamsters there was a trend for the plasma concentrations of both gonadotrophins (Fig. 2) to decline gradually during the 8-week period; however, this was not significant except that in the control group plasma FSH values were higher at Weeks 0 and 2 than at any other time $(P<0.01)$ and plasma LH concentrations at Week 8 were significantly lower $(P<0 \cdot 01)$. In contrast, the short-day hamsters showed dramatic changes in plasma gonadotrophin concentrations. In both the control and 6-MBOA-treatment groups plasma FSH values had fallen significantly $(P<0.01)$ within the first 2 weeks and were basal by Week 4. Plasma LH levels had also fallen significantly within the first 2 weeks, in both the control $(P<0.05)$ and 6-MBOA-treated group $(P<0.01)$, and were basal by Week 4 . Plasma gonadotrophin concentrations at Weeks 4-8 were clearly different in hamsters maintained under the two different photoperiods but chronic treatment with 6-MBOA had no effect on this photoperiodic response. The testes remained large in the hamsters which were maintained in long days and, as expected, regressed in hamsters which were transferred to short days (Fig. 1), closely following the decline in plasma gonadotrophin concentrations; after 4 weeks of short days testicular size had already decreased in the majority of the animals and by Week 6 all of the testes were non-palpable (i.e. approximately $400 \mathrm{mg}$ or less in weight). In spite of the dramatic differences in testicular weights, at Week 8 , between the long-day and short-day hamsters $(P<0.01)$, there were no differences between the control and 6-MBOA-treated groups (Table 1). Similarly, although long-day and short-day hamsters showed a significant difference in pituitary content of FSH $(P<0.05)$ and LH $(P<0.01)$, and mean serum testosterone concentrations $(P<0.01)$, there were no differences between the control and 6-MBOA-treated groups. Hypothalamic LHRH content and pituitary gland weight were identical in all four groups.

The rapid testicular regression shown by this inbred strain of hamsters (LSH/Ss Lak; Fig. 1) contrasts markedly with that shown by an outbred hamster strain (Lak:LVG (SYR)) which was obtained from the same commercial source (Charles River, Wilmington, MA, USA) and housed under identical environmental conditions. When hamsters from this latter strain were kept under 


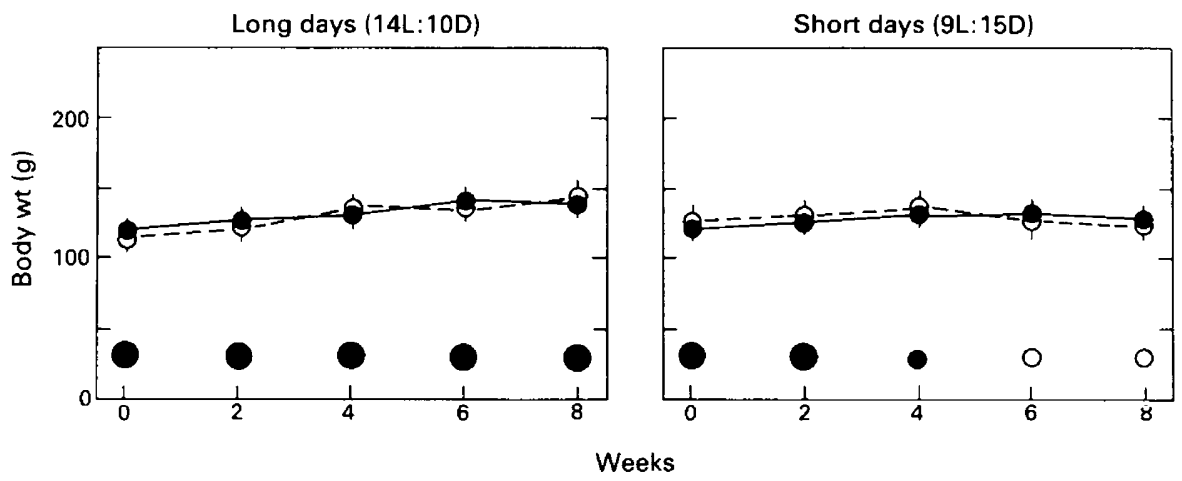

Fig. 1. Body weights of adult male hamsters implanted s.c. with empty (O-- $\mathrm{O})$ or 6-MBOAcontaining (-) elastomer capsules and maintained in long or short days. Each point represents a mean value and the s.e. are shown as vertical lines. Testicular size (width) was identical in 6-MBOA and control hamsters; pooled data are graphically represented by large solid circles $(>10 \mathrm{~mm})$, small solid circles $(6-10 \mathrm{~mm})$, and open small circles (completely regressed, non-palpable testes).

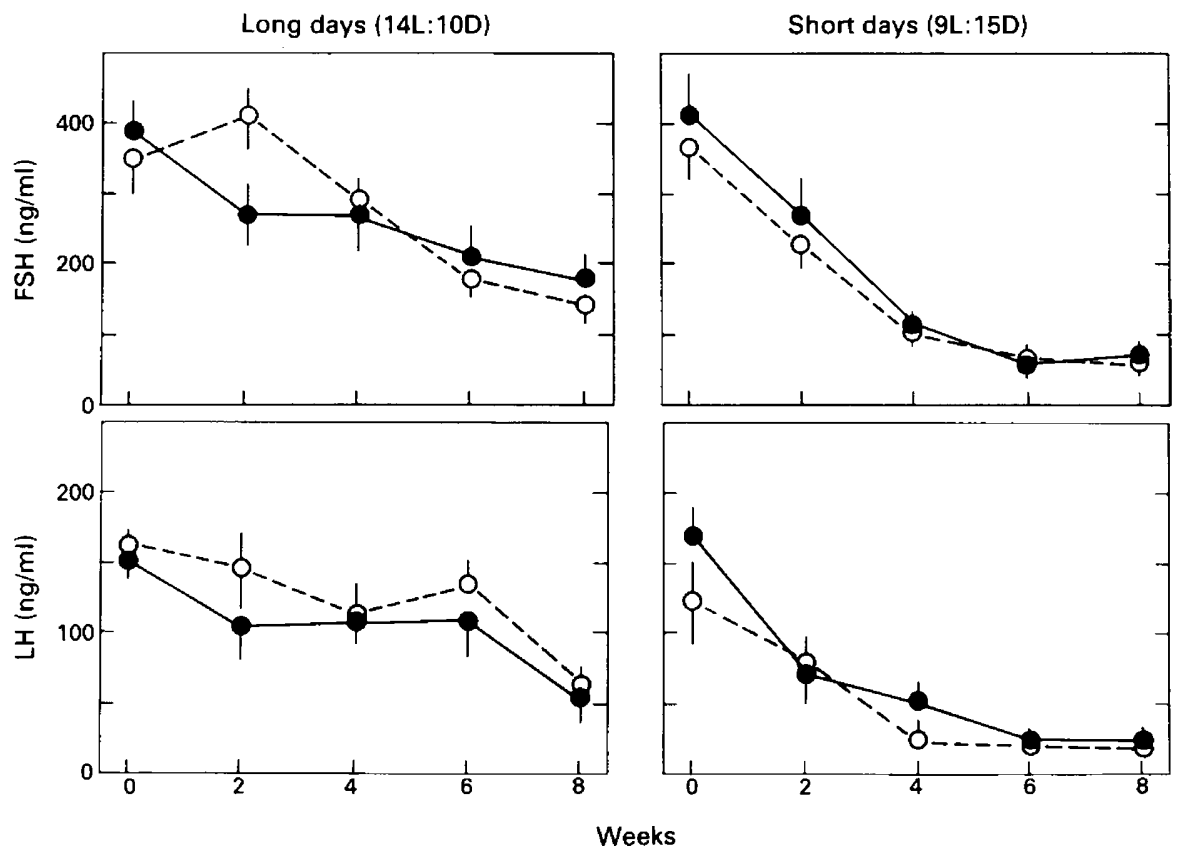

Fig. 2. Photoperiodic responses of adult male hamsters implanted s.c. with empty $\left(\mathrm{O}_{---} \mathrm{O}\right)$ or 6-MBOA-containing (-O) elastomer capsules. Each point represents a mean plasma FSH or LH value and the s.e. are shown as vertical lines.

long days their testicular diameter (measured through the scrotal wall) remained between 12 and $13 \mathrm{~mm}(\mathrm{~N}=14)$. In age-matched hamsters that were exposed to short days testicular diameter was still at this maximum level after 7 weeks $(\mathrm{N}=11)$ and reduced to $9.5 \mathrm{~mm}$ in only 1 animal; approximately 10 weeks of exposure to short days was required by this outbred strain for the testes to show complete regression. 
Table 1. Components of the hypothalamo-pituitary-gonadal axis of male hamsters maintained in long days or short days and treated with 6-MBOA for 8 weeks

\begin{tabular}{lccccc}
\hline & \multicolumn{2}{c}{ Long days } & & \multicolumn{2}{c}{ Short days } \\
\cline { 2 - 3 } \cline { 6 - 6 } & Controls & $6-\mathrm{MBOA}$ & & Controls & 6-MBOA \\
\hline Body weight $(\mathrm{g})$ & $142 \pm 4$ & $140 \pm 5$ & & $127 \pm 5^{*}$ & $129 \pm 4$ \\
Hypothalamic LHRH & $199 \pm 18$ & $173 \pm 17$ & & $197 \pm 23$ & $193 \pm 21$ \\
$\quad$ (pg/hypothalamus) & & & & \\
Pituitary gland wt (mg) & $4 \cdot 65 \pm 0 \cdot 13$ & $4 \cdot 6 \pm 0 \cdot 15$ & & $4 \cdot 38 \pm 0 \cdot 23$ & $4 \cdot 21 \pm 0 \cdot 21$ \\
Pituitary FSH $(\mu \mathrm{g} / \mathrm{gland})$ & $45 \cdot 5 \pm 5 \cdot 9$ & $50 \cdot 3 \pm 5 \cdot 6$ & & $25 \cdot 1 \pm 4 \cdot 5^{*}$ & $24 \cdot 7 \pm 9 \cdot 0$ \\
Pituitary LH $(\mu \mathrm{g} / \mathrm{gland})$ & $280 \pm 33$ & $288 \pm 29$ & & $88 \pm 17^{* *}$ & $103 \pm 14$ \\
Pituitary FSH $(\mu \mathrm{g} / \mathrm{mg})$ & $9 \cdot 8 \pm 1 \cdot 2$ & $11 \cdot 0 \pm 1 \cdot 4$ & & $5 \cdot 2 \pm 0 \cdot 7^{* *}$ & $6 \cdot 1 \pm 2 \cdot 3$ \\
Pituitary LH $(\mu \mathrm{g} / \mathrm{mg})$ & $60 \cdot 4 \pm 7 \cdot 3$ & $62 \cdot 6 \pm 6 \cdot 7$ & & $19 \cdot 2 \pm 3 \cdot 9^{* *}$ & $24 \cdot 5 \pm 3 \cdot 5$ \\
Testicular weight $(\mathrm{g})$ & $2 \cdot 68 \pm 0 \cdot 27$ & $2 \cdot 83 \pm 0 \cdot 10$ & & $0 \cdot 24 \pm 0 \cdot 04^{* *}$ & $0 \cdot 24 \pm 0 \cdot 04$ \\
Serum testosterone $(\mathrm{pg} / \mathrm{ml})$ & $2974 \pm 326$ & $3824 \pm 864$ & & $64 \pm 20^{* *}$ & $85 \pm 30$ \\
\hline
\end{tabular}

Values are means \pm s.e.

${ }^{*} P<0.05,{ }^{* *} P<0.01$ compared with Controls kept in long days.

\section{Discussion}

Studies of the neuroendocrine mechanisms that underlie mammalian photoperiodism depend upon the availability of model species which show a rapid and reliable photoperiodic response. In the present study the inbred male hamsters showed complete testicular atrophy within only 4-6 weeks of exposure to short days. This rapid gonadal regression was preceded by a marked decline in mean plasma concentrations of both LH and FSH, the first sign of a decrease occurring within just 2 weeks. A comparison between the reproductive axes of the long-day and short-day hamsters after 8 weeks revealed several differences. Testicular weight and serum testesterone concentrations were reduced by the short-photoperiodic exposure and, although the pituitary glands were the same weight under both photoperiodic treatments, the content of FSH and LH was significantly lower in the short-day groups. Since gonadotrophin production and release is controlled by LHRH one might have predicted a short-day induced decrease in hypothalamic LHRH content. However, this was not observed and is in general agreement with previously published reports of the content remaining unchanged or even increasing after extended periods of exposure to short daylengths (Pickard \& Silverman, 1979; Streger et al., 1982). It is therefore quite likely that reduced pituitary gonadotrophin content under short daylengths is primarily a reflection of attenuated LHRH secretion rather than a decrease in LHRH synthesis (Urbanski, 1990). Immunocytochemical examination of LHRH neuronal cell bodies in the diagonal band of Broca and medial preopticseptal brain areas of long-day and short-day hamsters, using a monoclonal LHRH antibody (Urbanski \& Hackett, 1989), has revealed very little difference in the number or staining intensity (Urbanski et al., 1990), thus further supporting this possibility.

The cyclic carbonate, 6-MBOA, has been shown to exert marked stimulatory effects on the reproductive axis of female and male microtine rodents (Berger et al., 1981, 1987; Sanders et al., 1981; Schadler et al., 1988). This non-oestrogenic compound, which is found in the leaves of some monocotyledonous plants may represent the primary environmental cue responsible for triggering the seasonal reproductive development in these species (Berger et al., 1977). It is not yet clear whether 6-MBOA can similarly affect the reproductive axis of other rodent species, especially those which are not strict vegetarians. It has been reported that the treatment of prepubertal female rats with Silastic capsules containing 6-MBOA, for 3 days, resulted in increased ovarian and uterine weights and increased serum FSH concentrations (Butterstein et al., 1985). Other investigators, however, have failed to observe this effect in spite of basically similar treatment (Vaughan et al., $1988 \mathrm{~b}$ ). We also have tried to affect reproductive development in female rats by implanting 22-day- 
old animals $(\mathrm{N}=10)$ with 6-MBOA-containing Silastic capsules. Such treatment had no effect on the age at which vaginal opening or first dioestrus occurred, relative to saline-treated controls $(\mathrm{N}=8)$; nor was there any difference in ovarian weight or the number of corpora lutea observed at first dioestrus (unpublished observations). Laboratory rats have been selectively bred for several decades and now show only weak responses to alterations in environmental cues such as photoperiod (Reiter, 1980); the same might also be true for responsiveness to 6-MBOA. The present study, therefore, used a highly photoperiodic strain of Syrian hamsters to test the effects of chronic 6-MBOA treatment on the hypothalamo-pituitary-testicular axis. It was proposed that such treatment might stimulate secretion of LHRH and in turn prevent or retard the gonadal regression that typically occurs under short photoperiods. In the male mountain vole 6-MBOA has been shown to cause testicular growth even when administered close to the winter solstice (Berger et al., 1981). The present results, however, fail to demonstrate any effect of 6-MBOA on the hypothalamo-pituitarytesticular axis of hamsters either under long days or after transfer to short days.

The rate of testicular regression under short daylengths and the decline in plasma LH and FSH was similar in the test and control animals. Similarly, examination of various reproductive measures after 8 weeks of exposure to short days failed to show any difference in body weight, hypothalamic LHRH content, pituitary gland weight and gonadotrophin content, testicular weight or serum testosterone concentrations. The route of administration of 6-MBOA adopted in this study was based on that previously reported by Butterstein et al. (1985). Although the possibility exists that an alternative route of administration or a higher dose would have been more effective in eliciting a vivid response within the hypothalamo-pituitary-gonadal axis, the findings of Anderson et al. (1988) do not support this view. In their investigation 6-MBOA was administered to hamsters by different routes, including daily injections, Silastic capsules, in the food, or in the drinking water. As in the present study, however, 6-MBOA had no effect on testicular size. Taken together, these findings complement those recently made when 6-MBOA was administered to hamsters that were maintained outdoors during the autumn (Vaughan et al., 1988a). It was found that the combined suppressive effect of short days and cold temperature on testicular size could not be overcome by the 6-MBOA treatment. Similarly, plasma LH and FSH concentrations and pituitary LH content were not affected although pituitary FSH content was significantly higher than in untreated controls. In summary, therefore, support for the hypothesis that 6-MBOA plays a physiological role in mediating the effect of seasonal changes in the environment on reproductive capacity in the male Syrian hamster is extremely weak. Whether or not the reproductive system of female hamsters is equally unresponsive to 6-MBOA is currently unknown.

This work (ORPRC No. 1717) was supported by NIH grants HD-24312, HD-18185 and RR-00163. We thank the NIDDK for the reagents used in the gonadotrophin radioimmunoassays; and Ms M. Nylund for her secretarial assistance. The LHRH fragments used in the specificity testing of antibody HU60 ([1-2]LHRH, [2-10]LHRH, [3-10]LHRH, [5-10]LHRH, [6-10]LHRH, [7-10]LHRH) and the pro-LHRH were gifts respectively, from Dr M. J. Kelly and Dr J. P. Adelman (both at the Oregon Health Sciences University, Portland, OR, USA); the [D-Phe ${ }^{2}$-LHRH] was a gift from Dr A. Arimura (Tulane University Hebert Center, Belle Chasse, LA, USA). All of the other peptides were obtained from Peninsula Laboratories, Inc. (Belmont, CA, USA).

\section{References}

Alleva, J.J. (1987) The biological clock and pineal gland: how they control seasonal fertility in the golden hamster. Pineal Research Reviews 5, 95-131.

Anderson, K.D., Nachman, R.Z. \& Turek, F.W. (1988) Effects of melatonin and 6-methoxybenzoxazolinone on photoperiodic control of testis size in adult male golden hamsters. J. Pineal Res. 5, 351-365.

Berger, P.J., Sanders, E.H., Gardner, P.D. \& Negus, N.C. (1977) Phenolic plant compounds functioning as reproductive inhibitors in Microtus montanus. Science, NY 195, 575-577.

Berger, P.J., Negus, N.C., Sanders, E.H. \& Gardner, P.D. (1981) Chemical triggering of reproduction in Microtus montanus. Science, NY 214, 69-70.

Berger, P.J., Negus, N.C. \& Rowsemitt, C.N. (1987) Effect of 6-methoxybenzoxazolinone on sex ratio and breeding performance in Microtus montanus. Biol. Reprod. 36, 255-260. 
Butterstein, G.M., Schadler, M.H., Lysogorski, E., Robin, L. \& Sipperly, S. (1985) A naturally occurring plant compound, 6-methoxybenzoxazolinone, stimulates reproductive responses in rats. Biol. Reprod. 32, $1018-1023$.

Gaston, S. \& Menaker, M. (1967) Photoperiodic control of hamster's testes. Science, $N Y$ 158, 925-928.

Hauser, U.E. \& Benson, B. (1986) Rapid cessation of estrous cyclicity and depressed castration response in short photoperiod-treated, inbred LSH/Ss Lak hamsters. Biol. Reprod. 35, 276-281.

Pickard, G.E. \& Silverman, A.J. (1979) Effects of photoperiod on hypothalamic luteinizing hormone releasing hormone in the male hamster. J. Endocr. 83, $421-428$.

Reiter, R.J. (1980) The pineal and its hormones in the control of reproduction in mammals. Endocr. Rev. 1, $109-131$.

Reiter, R.J., Sabry, I., Nordio, M., Vaughn, M.K. \& Migliaccio, S. (1989) Rate of reproductive involution following either exposure to short days or daily administration of melatonin is faster in inbred than in random-bred female Syrian hamsters. J. Endocr. 120, 489-496.

Resko, J.A., Malley, A., Begley, D. \& Hess, D.L. (1973) Radioimmunoassay of testosterone during fetal development of the rhesus monkey. Endocrinology 93, $156-161$.

Sanders, E.H., Gardner, P.D., Berger, P.J. \& Negus, N.C. (1981) 6-Methoxybenzoxazolinone: a plant derivative that stimulates reproduction in Microtus montanus. Science, $N Y$ 214, 67-68.

Schadler, M.H., Butterstein, G.M., Faulkner, B.J., Rice, S.C. \& Weisinger, L.A. (1988) The plant metabolite, 6-methoxybenzoxazolinone, stimulates an increase in secretion of follicle-stimulating hormone and size of reproductive organs in Microtus pinetorum. Biol. Reprod. 38, 817-820.

Steger, R.W., Bartke, A. \& Goldman, B. (1982) Alterations in neuroendocrine function during photoperiod induced atrophy and recrudescence in the golden hamster. Biol. Reprod. 26, 437-444.

Steger, R.W., Matt, K. \& Bartke, A. (1985) Neuroendocrine regulation of seasonal reproductive activity in the male golden hamster. Neurosci. Behav. Rev. 9, 191-201.

Stetson, M.H. \& Tate-Ostroff, B. (1981) Hormonal regulation of the annual reproductive cycle of golden hamsters. Gen. comp. Endocr. 45, 329-344.

Turek, F.W., Elliott, J.A., Advis, J.D. \& Menaker, M. (1975) The interaction of castration and photoperiod in the regulation of hypophyseal and serum gonadotropin levels in male golden hamsters. Endocrinology 96, $854-860$.

Urbanski, H.F. (1990) Activation of N-methyl-Daspartate (NMDA) receptors restores reproductive function in short-day Syrian hamsters. Endocrine Society Abstracts, Abstr. 1381.

Urbanski, H.F. \& Hackett, J. (1989) Development and characterization of monoclonal antibodies directed against LHRH. Neuroscience Abstracts 15, 1086.

Urbanski, H.F., Simpson, S.M., Ellis, D.H. \& Follett, B.K. (1983) Secretion of follicle-stimulating hormone and luteinizing hormone in castrated golden hamsters during exposure to various photoperiods and to natural daylengths. J. Endocr. 99, 379-386.

Urbanski, H.F., Doan, A. \& Pierce, M. (1990) Immunocytochemistry of LHRH neurons in hamsters exposed to different photoperiods. J. Reprod. Fert., Abstr. Ser. 5, Abstr. 89.

Vaughan, M.K., Little, J.C., Powell, D.C., Puig-Domingo, M. \& Reiter, R.J. (1988a) Melatonin and 6-methoxy-2benzoxazolinone (6-MBOA) alter the response of the male Syrian hamster to natural photoperiod. Int. $J$. Biometeor. 32, 103-107.

Vaughan, M.K., Little, J.C., Vaughan, G.M. \& Reiter, R.J. (1988b) Hormonal consequences of subcutaneous 6methoxy-2-benzoxazolinone pellets or injections in prepubertal male and female rats. J. Reprod. Fert. 83, 859-866.

Vendrely, E., Guérillot, C., Basseville, C. \& DaLage, C. (1971) Poids testiculaire et spermatogenèse du hamster doré au cours de cycle saisonnier. C. r. Séanc. Soc. Biol. Filiales 165, 1562-1565.

Received 7 December 1989 